\title{
Progress, Challenges and Perspectives in Positron Physics: Report on the XIIth International Positron Workshop
}

\author{
G. F. Gribakin ${ }^{1}$, H. Knudsen ${ }^{2}$ and C. M. Surko ${ }^{3}$ \\ ${ }^{1}$ Queen's University Belfast, Belfast BT7 1NN, United Kingdom \\ ${ }^{2}$ Department of Physics and Astronomy, University of Aarhus, DK 8000 Aarhus C, Denmark \\ ${ }^{3}$ Department of Physics, University of California at San Diego, La Jolla, CA, USA
}

Received January 28, 2004; accepted in revised form February 27, 2004

PACS Ref: $34.85 .+\mathrm{x}, 36.10 .-\mathrm{k}, 36.10 . \mathrm{Dr}, 71.60 .+\mathrm{z}$, 78.70.Bj, 07.77.-n, 41.75.Fr, 52.27.Ep.

\begin{abstract}
A report is presented of the XIIth International Workshop on Positron and Positronium Physics (Sandbjerg, Denmark, 19-21 July 2003). This workshop covered positron and positronium interactions with atoms, molecules and condensed matter systems. One key development reported was the first creation in the laboratory of low-energy antihydrogen atoms. Facets of positronelectron many-body systems were also considered, including the positronium molecule and BEC gases of positronium atoms. Aspects of the future of the field were discussed, including the development of new theoretical and experimental capabilities.
\end{abstract}

\section{Introduction}

This international positron workshop is traditionally held in conjunction with the biennial International Conference on Photonic, Electronic, and Atomic Collisions (ICPEAC). The 2003 conference was held at the beautiful Sandbjerg Estate, which is a conference center run by the University of Aarhus. This conference has a long history of focusing on forefront aspects of positron physics. This latest meeting had thirty invited talks and a comparable number of poster papers, many of which are described in the conference proceedings [1]. Topics of interest at the Workshop included positron and positronium collisions with atoms and molecules and multiple-positron systems, such as the $\mathrm{Ps}_{2}$ molecule. The possible Bose-Einstein condensation (BEC) of positronium atoms and new developments in positron sources and facilities were also discussed. A keen topic of discussion was the first production in the laboratory of low-energy antihydrogen atoms, which represents a crucial, first step in the creation and study of stable, neutral antimatter.

We first make a few introductory remarks about lowenergy positron physics. Typical sources of positrons are radioisotopes such as ${ }^{22} \mathrm{Na}$ and ${ }^{58} \mathrm{Co}$. Positrons can also be produced when high-energy electrons strike a high- $Z$ target, such as tungsten. In either case, the positrons have a broad spectrum of energies up to $\sim 1 \mathrm{MeV}$. They can be slowed to electron Volt energies through the use of "moderators," namely materials that have a positive work function for positrons, such as tungsten and solid noble gases (e.g., frozen neon). Sources of positrons are many orders of magnitude weaker (e.g., by a factor $\geq 10^{10}$ ) than corresponding electron sources, and this has been a great hindrance in studying positron-matter interactions experimentally.

Positrons can be detected by the gamma rays that are emitted when they annihilate with electrons. The lowest order decay processes are the emission of two $511 \mathrm{keV}$ gammas if the electron and positron are in an $S=0$ spin state, or three gammas if they are in an $S=1$ state. Positrons can also be detected using a channel electron multiplier (CEM) or micro-channel plate (MCP), or by directly collecting and measuring the charge.

Much interest is centered around the behavior of the neutral positronium atom (Ps), which is the bound state of an electron and positron (binding energy $(1 / 2) \mathrm{Ry}=6.8 \mathrm{eV})$. This atom is unstable to decay by annihilation. The lifetime of the $S=0$ state (para-Ps) is $0.125 \mathrm{~ns}$, while the lifetime of the $S=1$ state (ortho-Ps) is $143 \mathrm{~ns}$. The Ps atom can be detected by the characteristic two-gamma emission produced when it strikes a material surface or through use of a CEM or MCP.

\section{Antihydrogen}

A particularly significant event for antimatter physics was the recent creation of low-energy antihydrogen atoms. The experiments were conducted by two teams using the antiproton decelerator at CERN. Lars Jorgensen (University of Swansea, Swansea, UK) presented the results of the ATHENA collaboration [2], and Gerry Gabrielse (Harvard University, USA) described complementary results from the ATRAP team [3,4]. A long-term goal of this work is to make precise comparisons of the properties of antihydrogen and hydrogen in order to test CPT symmetry, namely symmetry with respect to charge conjugation, parity and time reversal, and to explore possible differences in the interaction of gravity with antimatter and matter. There are also many other interesting questions, such as the nature of collisions between antihydrogen and "ordinary" atoms (e.g., hydrogen and helium).

The source of slow antiprotons was the antiproton decelerator facility at CERN. The positron sources were ${ }^{22} \mathrm{Na}$. The two groups used similar techniques to combine cold clouds of antiprotons with cold positron plasmas. A schematic diagram and potential profile of the ATHENA nested trap is shown in Fig. 1. The clouds of antiprotons and positrons were trapped and cooled in a nested Penning trap, and then the antiprotons were forced through the trapped positron plasma. The experiments differed in the methods to accumulate the positrons. The ATHENA experiment accumulated $\sim 10^{8}$ positrons every 5 minutes in a buffer-gas trap, and then combined them with clouds 
(a) Csl crystals

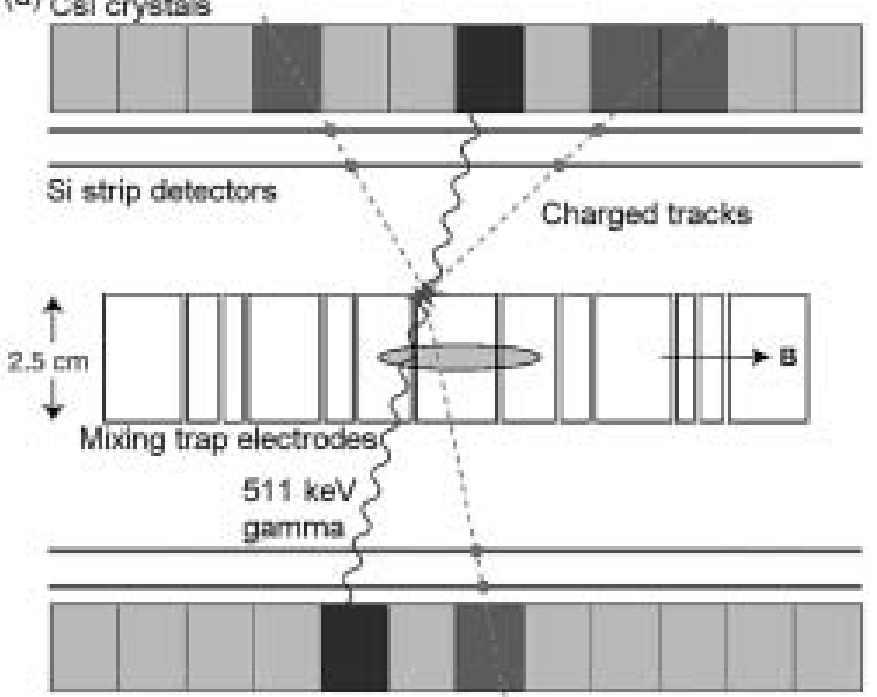

(b)

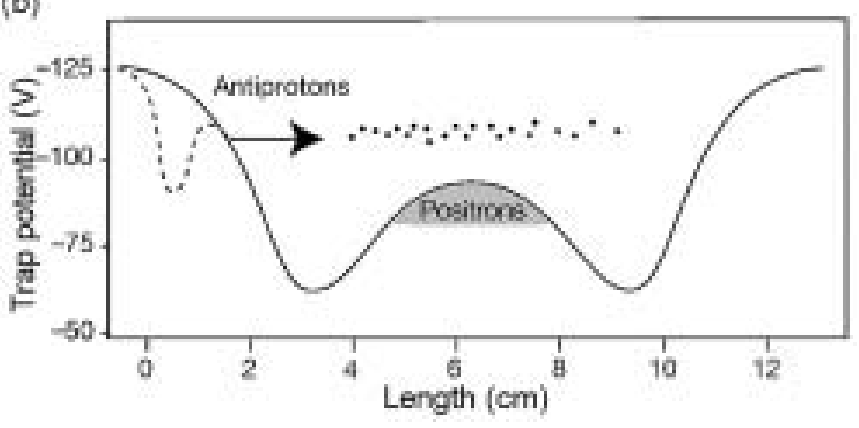

Fig. 1. Schematic diagram of the ATHENA nested trap and detector system: (a) The cylindrical electrodes and the positron cloud; also shown is a typical decay of an antihydrogen atom into three charged pions and two, back-to-back $511 \mathrm{keV}$ gammas; (b) Potential profile; the dashed line is the potential well for the antiprotons immediately before mixing, and the solid line is the potential during mixing. [Reprinted by permission from Nature, Ref. [2], copyright (2002), Macmillian Publishers, Ltd.]

of $\sim 10^{4}$ antiprotons. The ATRAP experiment accumulated positrons from high-Rydberg Ps atoms formed on the surface of a cold remoderator $\left(4 \times 10^{5}\right.$ positrons in $\sim 10 \mathrm{~min}$. ) and combined them with clouds of up to $4 \times 10^{5}$ antiprotons.

The methods to detect the antihydrogen atoms were also different in the two experiments. In the ATRAP experiment, the antihydrogen was field-ionized in the vicinity of a potential well that was specifically arranged to trap the resulting antiprotons, which were then dumped and detected. The ATHENA collaboration built an antihydrogen detector that is able to resolve in space and time the annihilation of the neutral antihydrogen atoms that, after formation, move across the magnetic field and strike the electrodes of the Penning trap. This detector, which is shown schematically in Fig. 1(a), is able to resolve both the vertex of the shower of mesons from the antiproton decay and the back-to-back $511 \mathrm{keV}$ gamma rays from the positron annihilation. When the antihydrogen production rate was extrapolated for the angular acceptance and efficiency of the detection techniques, the results of both experiments indicated that surprisingly large amounts of antihydrogen had been produced (e.g., $\geq 10^{6}$ atoms).

It is believed that antihydrogen in these experiments can be formed by two different processes, either by sponta- neous emission of a photon in a positron-antiproton collision, or three-body recombination involving two positrons and an antiproton. The ATRAP experiment was able to estimate the binding energy of the antihydrogen from their field-ionization data. They concluded that the atoms in their experiment are weakly bound (i.e., by $\sim 1 \mathrm{meV}$ ), which is consistent with the three-body process.

The ATHENA group was able to measure the dependence of the antihydrogen formation rate, $\Gamma_{\mathrm{f}}$, on positron temperature, by heating the positron plasma with an $\mathrm{rf}$ signal, and measuring the resulting change in $\Gamma_{\mathrm{f}}$. A preliminary analysis of the data indicates that $\Gamma_{\mathrm{f}} \sim 1 / T$ [5]. In the spontaneous photoemission process, $\Gamma_{\mathrm{f}}$ is expected to scale as $1 / T$, while for the three-body process, it is expected that $\Gamma_{\mathrm{f}} \sim 1 / T^{9 / 2}$. Surprisingly, the ATHENA group found an appreciable formation rate even at $T=300 \mathrm{~K}$.

The ATHENA and ATRAP results are the beginning of a new day in antimatter physics, raising a myriad of interesting questions and opening the door to many scientific opportunities. The antihydrogen atoms are expected to be weakly (moderately weakly) bound in both formation processes; $\sim 1 \mathrm{meV}$ in the three-body process, and much more tightly bound for the spontaneous, photon emission process. It will be important to understand the production mechanisms in detail; and explore, for example, the role that stimulated photorecombination (using an external radiation source) might play in enhancing the efficiency of antihydrogen production, particularly to deeply bound states. There is also an important issue regarding the nature and dynamics of the high-Rydberg antihydrogen atoms in the electric fields of the single component positron plasma and the ambient several-tesla magnetic field. The positron is localized to a region small compared to the antiproton-positron separation distance, and so the antihydrogen atoms are most properly regarded as "guiding-center atoms" that move by classical $\mathbf{E} \times \mathbf{B}$ drift dynamics [6]. The dynamics and fate of these atoms in the electric fields of the trap and the combining plasmas is a key question for antihydrogen physics. There are also questions regarding possible instabilities of the combining plasmas.

Beyond the antihydrogen formation process, there is the quest to make precision measurements on antihydrogen. One approach to comparing hydrogen and antihydrogen involves cooling and trapping these antiatoms. The natural choice is to trap the neutral atoms in a magnetic-gradient trap (well depth $\leq 1$ kelvin). Unfortunately, this does not appear to be compatible with the nested Penning traps used to confine the antiprotons and positrons [7]. While several schemes have been proposed to circumvent this problem, finding the best method to trap and further cool antihydrogen remains an open question. Measurement of the spin magnetic moment in a beam geometry (which would avoid the need to trap the antihydrogen atoms) is also being pursued [8].

The antihydrogen experiments bring to the fore a number of interesting positron physics problems. The ATRAP technique to make high-Rydberg positronium atoms from a cold remoderator is of interest for other applications, such as precision measurements on positronium. In addition, in work led by Eric Hessels (York 
University, Toronto, Canada), the ATRAP group has prepared a source of cold high-Rydberg positronium atoms (made via charge exchange of positrons on Cs atoms laserprepared in high Rydberg states). This positronium beam will be used, in turn, to prepare high-Rydberg antihydrogen by charge-exchange [9]. If successful, this would be another way of preparing antihydrogen atoms which, in this case, are all in the same state.

In another talk at the conference, Robert Bluhm (Colby College, Waterville, USA) discussed tests of Lorentz and CPT invariance, including tests in antihydrogen [1]. Violation of these symmetries is a feature of some field theories incorporating gravity. It takes place at the Planck mass scale, $M_{\mathrm{Pl}}=(h c / G)^{1 / 2} \approx 10^{19} \mathrm{GeV}$, and so its effect at "normal" energy scales is greatly suppressed. A recent paper, by $\mathrm{O}$. W. Greenberg presents a proof that CPT violation leads to violation of Lorentz invariance [10]. High-precision atomic spectroscopic measurements of the forbidden 1S-2S transition in hydrogen and antihydrogen could, at least in principle, detect such small effects. An alternative to studying the $1 \mathrm{~S}-2 \mathrm{~S}$ transition would be measurement of the ground-state hyperfine splitting. This would provide a bound on a CPT-violating parameter for the proton.

There are many open questions regarding the interaction of neutral antimatter and matter. This is of considerable practical significance, since the ability of antihydrogen to survive even a few collisions with matter could potentially provide an important method to cool these antiatoms to lower temperatures. At present, antihydrogen-matter collisions are being considered theoretically by several groups. The simplest neutral antimatter-matter interaction, collision of antihydrogen with hydrogen, has been considered by a number of groups. Edward Armour (University of Nottingham, Nottingham, U.K.) discussed calculations by his group for this system, including elastic and rearrangement channels. In particular, he discussed a disagreement between the cross sections for the formation of protonium in Rydberg states with $n=22,23,24$ obtained by different groups [1]. He also discussed calculations for the interaction of helium and antihydrogen. In this system he described an interesting structure in the (Born-Oppenheimer) potential that exhibits a minimum at $3.6 a_{0}$ and a maximum at 2.4a $a_{0}$. Arnab Ghosh (Indian Association for the Cultivation of Science, Kolkata, India) reported the results obtained by their group for close-coupling calculations of collisions of antihydrogen with hydrogen and helium [1]. Their best prediction of the antihydrogenhelium scattering length is $2.747 a_{0}$. There is, however, some recent evidence that this quantity may in fact be strongly affected by the strong antiproton- ${ }^{4} \mathrm{He}$ interaction [11].

\section{Positron collisions}

3.1 Low energies: elastic scattering, target excitation, and annihilation

Much recent progress in the area of positron collisions with atoms and molecules has been achieved in the difficult low and low-to-medium energy ranges. On the experimental side, a breakthrough was made with the advent of a trapbased highly monochromatic positron beam [12]. Positrons are accumulated and cooled to ambient temperature (i.e., $\sim 300 \mathrm{~K}=25 \mathrm{meV}$ ) in a Penning-Malmberg trap by collisions with a dilute gas of $\mathrm{N}_{2}$ molecules. They can then be gently forced out of the trap to form a positron beam with energy resolution more than a factor of 10 smaller than typical beams used previously in atomic physics experiments. This development has, for example, enabled the first measurements of the energy dependence of annihilation rates for positron interactions with atoms and molecules.

The annihilation rate is conventionally expressed in terms of the parameter, $Z_{\text {eff }}$, which is the annihilation rate normalized to that for a gas of free electrons at electron density $n_{\mathrm{e}}=n_{\mathrm{m}}$, where $n_{\mathrm{m}}$ is the density of molecules. Thus, neglecting correlations, for molecules with a total number of electrons $Z$, it would be expected that $Z_{\text {eff }}=Z$. A longstanding puzzle is that for certain species, such as large hydrocarbons, one finds $Z_{\text {eff }} \gg Z$. The quantity $Z_{\text {eff }}$ has now been measured for positron energies from $50 \mathrm{meV}$ to several electron-volt with the energy resolution $\leq 25 \mathrm{meV}[13,14]$. Data for butane $\left(\mathrm{C}_{4} \mathrm{H}_{10}\right)$ are shown in Fig. 2. These experiments have shown that the very large values of $Z_{\text {eff }}$ observed for large polyatomic molecules (e.g., alkanes) are due to positron capture in vibrational Feshbach resonances (VFR). This topic was discussed at the conference by G. Gribakin and P. Gill (Queen's University, Belfast; and University of Nottingham, UK) [1].

The experiments support the theoretical predictions that VFR play a crucial role in positron annihilation on molecules with positive positron affinities [15]. They also provided the first evidence that many polyatomic molecules are capable of forming bound states with positrons and gave estimates of the binding energies $(\sim 33 \mathrm{meV}$ in the case of butane, as obtained from the data in Fig. 2). This concerted theoretical and experimental effort has led to a considerable advance in solving the 50-year-old puzzle of anomalously high positron annihilation rates in many polyatomic gases $[16,17]$. However, many questions about the details of this phenomenon remain to be addressed.

At the meeting, Levi Barnes (University of California, San Diego, USA) presented an overview of the experimental data on energy-resolved annihilation on molecules $[1,13,14]$. The most prominent feature in the data for

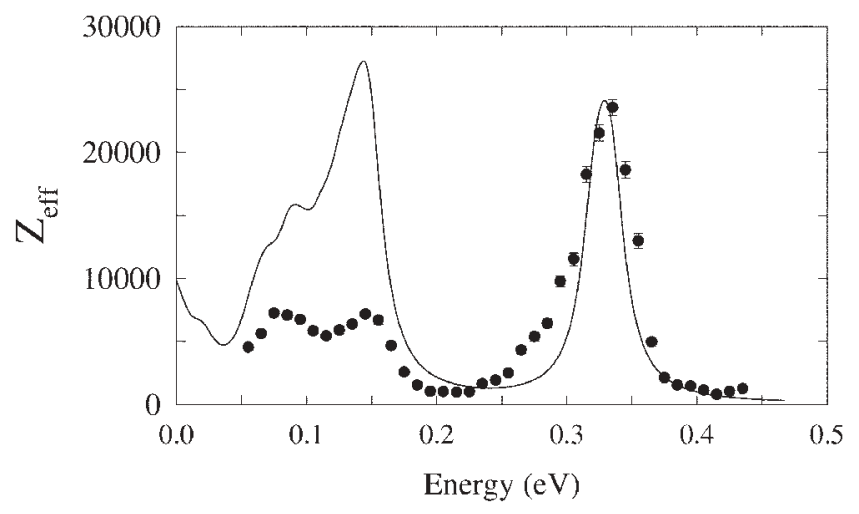

Fig. 2. The energy dependence of $Z_{\text {eff }}$ for butane $\left(\mathrm{C}_{4} \mathrm{H}_{10}\right)$ measured with the trap-based positron beam (solid circles) [13,14]. Also shown is a fit (solid line) to a sum of Breit-Wigner profiles (FWHM $25 \mathrm{meV}$ ) centered on the fundamental vibrational modes of the molecule, downshifted by $33 \mathrm{meV}$ to fit the position of the $\mathrm{CH}$ stretch peak $[1,14]$. 
alkanes are peaks at positron energies corresponding to those of the $\mathrm{CH}$ stretch vibrations, such as that shown in Fig. 2. Observations of a systematic downshift of the peaks yield estimates of the positron-molecule affinities, ranging from $30 \mathrm{meV}$ in butane to $200 \mathrm{meV}$ in dodecane $\left(\mathrm{C}_{12} \mathrm{H}_{26}\right)$. Barnes also reported the results for the energy dependence of $Z_{\text {eff }}$ in $\mathrm{Ar}$ and $\mathrm{Kr}$, where the low-energy values of $Z_{\mathrm{eff}}$ are enhanced by the presence of positron-atom s-wave virtual levels, and data on the effect of fluorination on the $\mathrm{CH}$ stretch peaks observed in alkanes. Substitution of even a single hydrogen atom with a fluorine in hexane and nonane leaves the position of the $\mathrm{CH}$ stretch peak unchanged, but markedly reduces its magnitude. The data for methane and its fluorine substituted analogues also show rapid changes with the number of fluorine atoms. These new experimental results pose many new questions and will no doubt stimulate future theoretical work in the area.

A full theoretical treatment of positron-molecule collisions, including molecular vibrations (and rotations) remains a difficult problem. The methods applied to date either treat the electron-positron correlations $a b$ initio, leaving out vibrations, or use a model-potential approach to describe the positron-molecule interaction and include vibrations by means of a close-coupling method. An example of the first type of calculation was reported by Marco Lima, P. Chaudhuri and collaborators (Universidade Estadual de Campinas, Sao Paulo, Brazil) [1]. He and his collaborators used a Schwinger multichannel method to calculate positron scattering from the nitrogen molecule. They focused on the excitation cross section of the $a^{1} \Pi_{g}$ state, for which the experiment [18] shows a rapid rise from threshold, possibly indicating a shape resonance. In spite of the earlier success of this method in treating low-energy $(\sim 1 \mathrm{eV})$, elastic scattering from $\mathrm{H}_{2}, \mathrm{CH}_{4}, \mathrm{~N}_{2}, \mathrm{C}_{2} \mathrm{H}_{2}$ and $\mathrm{C}_{2} \mathrm{H}_{4}$, the presence of spurious resonant structures [1] prevented the authors from verifying the nature of the effect in nitrogen. The difficulty of the problem may be related to the fact that the $\mathrm{a}^{1} \Pi_{\mathrm{g}}$ excitation lies above the threshold for positronium formation, a process that has, thus far, been beyond the capacity of the Schwinger multichannel method.

In a calculation of the second type, discussed by Franco Gianturco (University of Rome La Sapienza, Rome, Italy), an attempt was made to study the dependence of the energy of a virtual level or weakly bound positron state on molecular geometry [1]. Their method employs a fixednuclear orientation approximation and represents the interaction between the positron and molecular target by a local model potential, which consists of the electrostatic and correlation-polarization terms. The annihilation parameter $Z_{\text {eff }}$ is found as an overlap between the ground-state electron density and the single-particle positron density. In earlier work, these approximations were used by the authors to calculate elastic and vibrational excitation cross sections and $Z_{\text {eff }}$ for a number of molecules. While good agreement was obtained in a number of scattering calculations (e.g., $\mathrm{H}_{2}, \mathrm{CO}, \mathrm{NH}_{3}, \mathrm{CH}_{4}, \mathrm{CF}_{4}, \mathrm{C}_{2} \mathrm{H}_{2}$ ) [1], the results for $Z_{\text {eff }}$ show little correlation with the experimental data, especially for polyatomic molecules.

The calculations reported at the workshop show that molecules like $\mathrm{C}_{2} \mathrm{H}_{2}$ and $\mathrm{C}_{2} \mathrm{H}_{4}$ possess positron virtual states. These states are caused by the attractive polarization-correlation potential and are a common feature of many larger atoms (e.g., Ar and $\mathrm{Kr}$ ) and other easily polarizable targets. Forty years ago Gol'danskii and Sayasov [19] pointed out that positron virtual or weaklybound s-type states should lead to enhanced values of $Z_{\mathrm{eff}}$ at low energies. This enhancement was demonstrated in molecules by the calculation of Lima et al. (for $\mathrm{C}_{2} \mathrm{H}_{2}$ and $\mathrm{C}_{2} \mathrm{H}_{4}$ ), although they failed to reproduce experimental values of $Z_{\text {eff }}$. The work done in Rome shows that, if the geometry of the molecule is distorted by either bending or stretching, a virtual level can turn into a weakly bound state. It is conjectured that such processes might provide a path for populating VFR. It is fair to say that the problem of VFR in low-energy electron- and positron-molecule collisions and their role as mediators of various reactions (e.g., positron annihilation) is drawing considerable attention from both theorists and experimentalists.

An important advance in the theory of low-energy scattering and annihilation on many-electron atoms, was reported in poster papers by John Ludlow (Queen's University, Belfast, UK). In this work a many-body theory approach was developed to include accurately the two main correlation effects that contribute to the positronatom attraction, namely target polarization and virtual positronium formation. The use of B-splines and extrapolation over the angular momenta of the electron and positron orbitals allowed the group to achieve converged results for the scattering phase shifts and for a much more sensitive annihilation parameter $Z_{\text {eff }}$. The calculations demonstrate very good agreement for the total and differential elastic cross sections and annihilation rates for the noble gases. In particular, the theory confirmed the high measured value $[20,21]$ of $Z_{\text {eff }} \approx 400$ for roomtemperature positrons on Xe.

John Humberston (University College London, London, UK) discussed the scaling of Ps-formation cross sections with the Ps-formation threshold energy, $E_{\mathrm{Ps}}$, of the form $\sigma_{\mathrm{Ps}}=A \exp \left(-B E_{\mathrm{Ps}}\right)$, where $A$ and $B$ are target-independent functions of excess energy. As a possible way of establishing $A$, the results of a calculation for a model atom with the $E_{\mathrm{Ps}}=0$, i.e., ionization potential of $6.8 \mathrm{eV}$, were analyzed.

\subsection{Medium energies: positronium formation and ionization}

A talk by Walter Kauppila (Wayne State University, Detroit, USA) summarized the achievements of the Detroit group in measuring total and differential positron $\left(\mathrm{e}^{+}\right)$ scattering cross sections and positronium-formation cross sections (i.e., the reaction, $\mathrm{e}^{+}+A \rightarrow A^{+}+$Ps) for a range of atomic and molecular targets at positron energies from one to hundreds of eV. Poster presentations by E. Surdutovich and collaborators (Wayne State University, Detroit, USA) reported related work, an extension of the positron-alkali atom scattering studies to Cs [1]. The Cs atom has the lowest ionization potential of any alkali atom studied to date and is expected to have the highest fraction of excited state positronium formation. The experimental data for the total Ps formation cross section are in reasonable accord with the coupled-state calculations [22]. However, a comparison of the results for $\mathrm{K}, \mathrm{Rb}$ and $\mathrm{Cs}$ shows that the measured "lower-limit" cross section decreases along the sequence, while the theory predicts the opposite. From an 
experimental point of view, the difficulty lies in detecting all Ps annihilation gamma quanta.

An interesting new development is an experiment to measure both $2 \gamma$ and $3 \gamma$ annihilation gamma quanta (the latter resulting from the decay of ortho-Ps). As shown in Fig. 3, the energy dependence of the ratio of the $3 \gamma$ and $2 \gamma$ signals, from experiments by Bert Stein and collaborators, appears to depend on the energies of the Ps atoms formed [1]. These experiments have the potential to yield information about ortho-Ps surface interactions, and possibly on positronium formation by electrons from inner orbitals. An intriguing result from these experiments is that, for $\mathrm{CH}_{4}$, the $3 \gamma$ to $2 \gamma$ signal provides evidence that the threshold for Ps formation is determined by the adiabatic ionization energy, rather than a vertical one.

Joan Marler (University of California, San Diego, USA) reported the results of absolute measurements of the Ps-formation and ionization cross sections in $\mathrm{Ar}, \mathrm{Kr}$ and Xe with a trap-based positron beam $[1,23]$. Data for Ar and comparison with other measurements and theory are shown in Fig. 4. Comparison of the San Diego data with the distorted-wave Born calculations by Sharon Gilmore, et al. [1] indicates that, although the theoretical cross sections are similar in shape, their absolute magnitudes differ by a factor of two to three. The cross sections measured by the San Diego group are close to those obtained previously at UCL using a different method, namely subtracting from the total ion yield the yield due to direct ionization [24]. However, the San Diego data did not show the double-peak structure, which was especially prominent in the UCL data for Ar. To understand this discrepancy, the direct ionization cross section measured with the trap-based beam for Ar from threshold to $80 \mathrm{eV}$, was subtracted from the UCL total ionization cross section. The Ps-formation cross section thus obtained is in much better agreement with the absolute data measured in the trap, indicating a possible deficiency in the earlier [24] direct ionization data.

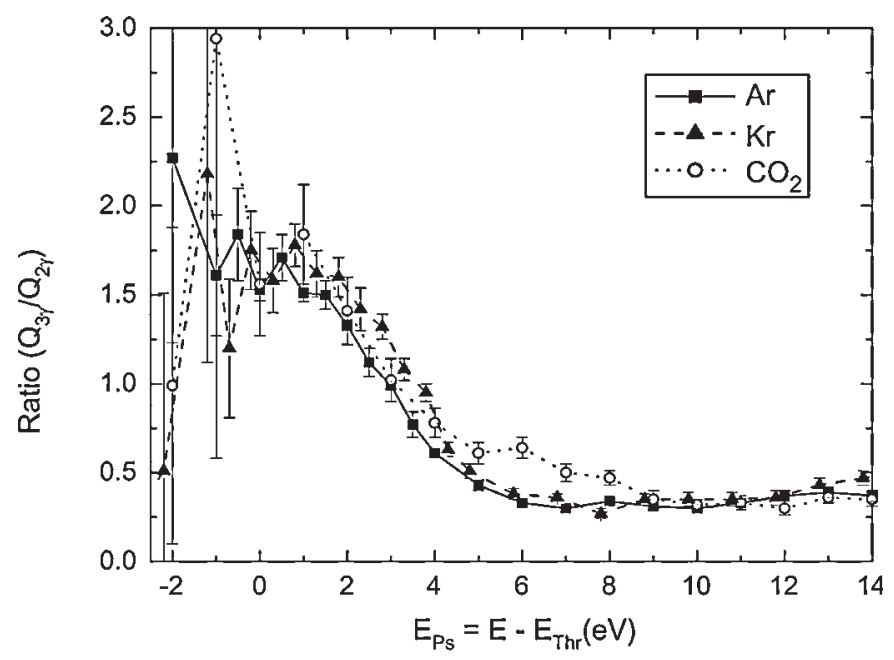

Fig. 3. Ratio of three-gamma to two-gamma emission due to positronium formation in positron collisions with $\mathrm{Ar}, \mathrm{Kr}$ and $\mathrm{CO}_{2}$, plotted as a function of positronium kinetic energy, E_Ps, which is defined as the incident positron energy minus the threshold energy for positronium formation. The three-gamma emission is due to in-flight decay of orthoPs, and the two-gamma emission is due to para-Ps decays and annihilation of ortho-Ps at the walls of the gas cell. See T. S. Stein, et al., Ref. [1].

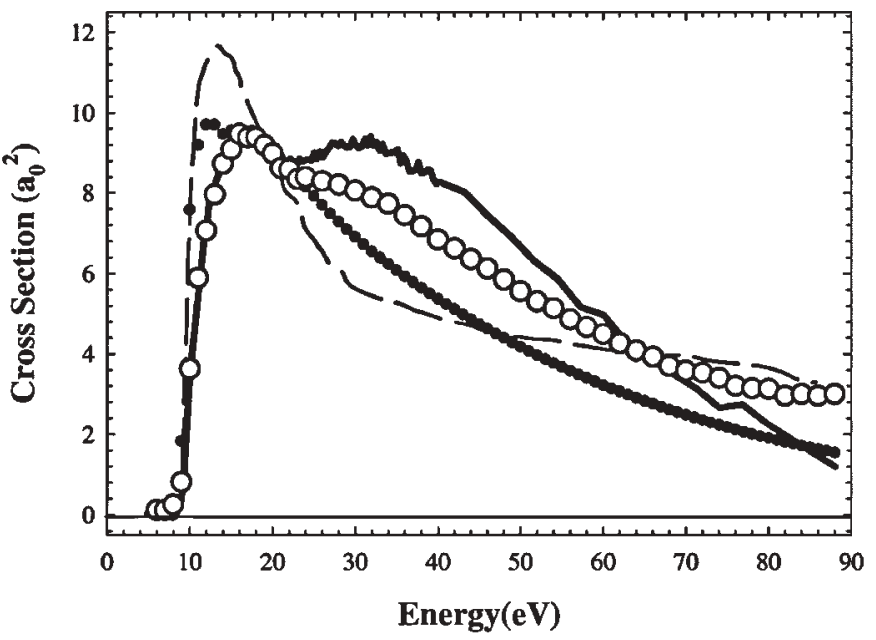

Fig. 4. Absolute positronium formation cross section measurements for positrons on argon: $(\bigcirc)$ [33]; (-) [24]. The data are in good, absolute agreement except for a possible second peak in the data of Ref. [24] in the range $\sim 30-60 \mathrm{eV}$. Both data sets are consistent with upper and lower limits on the cross section measured in a third experiment [34]. Also shown are the predictions of theory of Ref. [35] (- ), and a more recent calculation

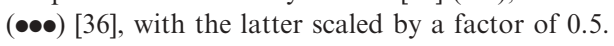

New measurements of the Ps formation cross sections for $\mathrm{Ar}$ and $\mathrm{Xe}$ were reported by Marta Szluinska and Gaetana Laricchia (University College London, UK) [1]. In this work, the total ion yield was measured in coincidence with the annihilation $\gamma$ rays, and normalized to the total ionization cross section. The results obtained by this method agree with the earlier Ps formation data from UCL (although they tend to be higher at larger positron energies). In particular, these measurements confirm a double-peak shape of the cross section measured previously by the UCL group. The origin of this structure is still unclear. While the position of the 2 nd peak is close to the ionization threshold of the first sub-valence electron, its onset is near to the threshold for Ps-formation involving this state. This suggests that the structure may be due to Ps formation on sub-valence electrons. Another suggestion is that the peak is due to Ps formation in excited states [24]. A theoretical attempt to test this hypothesis was reported by Sharon Gilmore (Queen's University, Belfast, U.K.) [1]. The calculations do show an onset due to Ps formation in excited states. However, the relative size of this component is smaller than that observed in the UCL experiment, and the energy where it occurs is considerably lower than seen in experiment. In summary, comparing the UCL and San Diego data, the existence of the "double-peak" structure is not resolved. Assuming it does exist, the origin of this feature is unclear.

Akos Kover (Institute of Nuclear Research of the Hungarian Academy of Science, Debrecen, Hungary) reported the results of an experimental study of electron capture to the continuum (ECC) in positron-atom/molecule ionization. The ECC effect manifests itself as a peak in the electron energy distribution measured in the forward direction at the energy when the electron velocity matches that of the final-state positron. The experimental data for $100 \mathrm{eV}$ positrons on $\mathrm{H}_{2}$, which show such a structure, are consistent with the theoretical predictions [25]. However, the peak in the electron spectrum obtained with $50 \mathrm{eV}$ positrons 
appears to be downshifted by $1.6 \mathrm{eV}$. Currently, there are no quantum calculations that would explain this effect.

\section{Positronium, Ps-atom collisions and many-Ps effects}

\subsection{Ps scattering from atoms}

A comprehensive overview of Ps-atom scattering experiments was presented by Gaetana Laricchia (University College London, UK). Her presentation spanned a range of problems, from the creation of a Ps beam to the studies of the total scattering cross sections of Ps by atoms and molecules and Ps break-up in collisions. In particular, the data from UCL show that, contrary to earlier expectations, most of the atoms in Ps beams formed from Xe and $\mathrm{H}_{2}$ targets are in the ground state. Another interesting observation is that Ps-beam formation in Xe is surprisingly ineffective, in spite of a large Ps-formation cross section. One explanation links this effect to a broad angular distribution of the Ps formed from Xe.

Comparisons were made of the measured Ps-atom scattering cross sections for $\mathrm{H}_{2}, \mathrm{He}$ and $\mathrm{Ar}$ with various theories from threshold to about $100 \mathrm{eV}$. They show that, for a given target, the results obtained by different methods agree to within a factor of two. This is illustrated in Fig. 5 for Ps scattering from Ar. However, experimental estimates of the forward-scattering, Ps-Ar differential cross sections in the range $15-100 \mathrm{eV} \mathrm{[26]} \mathrm{are} \mathrm{a} \mathrm{factor} \mathrm{of} \mathrm{ten} \mathrm{larger} \mathrm{than} \mathrm{the}$ values calculated in Ref. [27] at $5 \mathrm{eV}$. This important subject warrants further theoretical and experimental work.

Positronium scattering from atoms has been considered theoretically by several groups. At the meeting James Walters (Queen's University, Belfast, U.K.) presented the results obtained using a coupled pseudostate approach, which extends over several tens of electron Volts. At very low energies, calculations of Ps-atom scattering were done recently by adapting an essentially bound-state stochastic variational method, which allows for an accurate account of few-body correlation effects (e.g., dynamic distortion of positronium by the atom) [28]. However, at present, there is still little accord between various theories and a range of

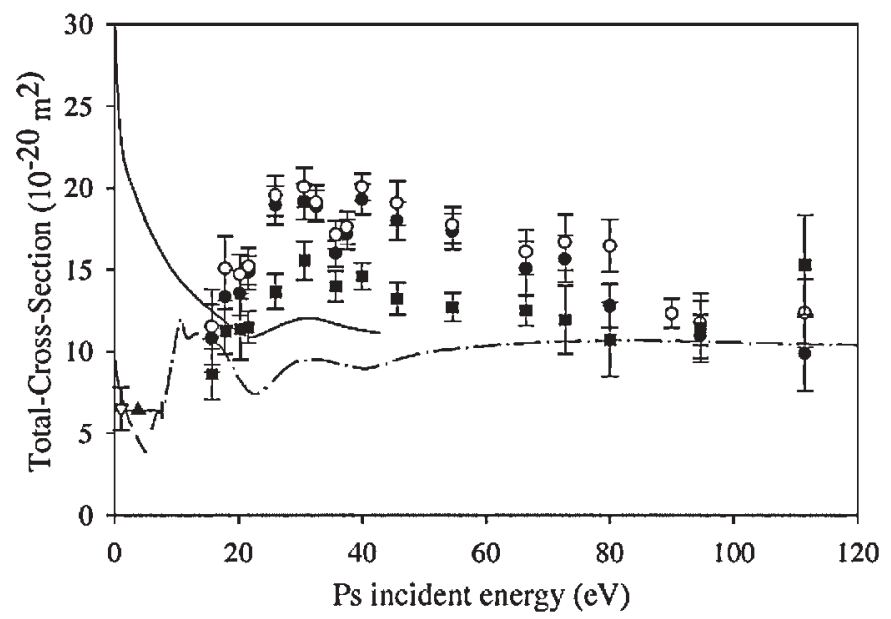

Fig. 5. Total cross-section measurements and theoretical calculations for Ps scattering from argon. Experiment: (hollow circles), $\Theta \sim 0^{\circ}$, Garner et al. [26]; (full circles) $\Theta \sim 1.5^{\circ}$, Garner, et al. [37]; (full squares) $\Theta \sim 6^{\circ}$ Zafar et al. [38]; (full triangle) Skalsey et al. [39]; (hollow triangle) Coleman et al. [40]. Theory: (solid line) Blackwood et al. [41]; (dashed line) Biswas and Adhikari [42], (dash-dot line) McAlinden et al. [43]. experimental data based on measuring the Ps-atom momentum-transfer cross sections (i.e., done by detecting the two annihilation $\gamma$ quanta from ortho-para Ps conversion).

An important problem in low-energy Ps-atom collision physics is annihilation. The lifetime of ortho-Ps in a gas depends on the probability of pick-off annihilation that leads to an immediate annihilation into two gammas. Another process that depletes ortho-Ps is ortho-to-para conversion. This process can take place in collisions with atoms or molecules with an unpaired electron (e.g., $\mathrm{O}_{2}$ ), or due to the spin-orbit interaction of the constituents of Ps in the atomic field (see Section 4.3).

\subsection{Ps and $P_{S_{2}}$}

A number of interesting questions regarding the physics of Ps and the positronium molecule, $\mathrm{Ps}_{2}$, were discussed at the conference. Lars Madsen (University of Aarhus, Denmark) described calculations of the kinds of Ps states that can be accessed by laser excitation [1]. Topics discussed included the creation of ensembles of Ps with substantial excitedstate populations and aspects of Ps spectroscopy. Also discussed was the possibility of forming antihydrogen by laser-assisted charge exchange between Ps and antiprotons.

U. Uggerhoj (University of Aarhus, Denmark) discussed planned experiments to study the multiphoton ionization of Ps and the photo-detachment of $\mathrm{Ps}^{-}$, and D. Schwalm (Max-Planck-Institut für Kernphysik, Heidelberg, Germany) described an experiment to study the decay rate of the $\mathrm{Ps}^{-}$negative ion [1]. Finally, Y Suzuki (Niigata University, Niigata, Japan) described calculations of the excited states and resonances of the $\mathrm{Ps}_{2}$ molecule [1].

\subsection{Many-Ps effects}

Work in this area follows early suggestions of interesting many-Ps effects [29] and ranges from a first observation of the $\mathrm{Ps}_{2}$ molecule to the quest for a BEC gas of Ps atoms, and the possibility of observing stimulated annihilation radiation at $511 \mathrm{keV}$. A theoretical overview of the quantum, many-positron many-electron system was presented by Hiroyuki Yabu (Tokyo Metropolitan University, Tokyo, Japan) [1]. He discussed the critical role of Ps-Ps scattering lengths in determining the phase diagram. He described a phase diagram with $\mathrm{Ps}_{2}$ molecules, a BEC phase, and an electron-positron liquid (possibly as a BCS phase), in addition to the classical electron-positron plasma at high densities and temperatures.

A BEC Ps will require a gas of spin-polarized Ps atoms. Toshio Hyodo (University of Tokyo, Tokyo, Japan) and colleagues have been working on understanding Ps spin dynamics. They reported on experiments to study ortho-topara spin conversion of Ps atoms in spin-exchange collisions with $\mathrm{O}_{2}$ (i.e., molecules with unpaired electron spins). They reported a cross section of $3 \times 10^{-19} \mathrm{~cm}^{2}$, which is about 100 times greater than that expected from pick-off. In related work, Mitroy and Novikov [30] recently considered the possible role of the spin-orbit interaction in converting ortho-Ps into para-Ps in quasi-elastic collisions. This latter process requires a p-wave collision. As a consequence, this mechanism has a strong energy dependence. It provides a possible explanation of the dearth of thermal Ps observed in positron lifetime experiments with 
$\mathrm{Kr}$ and $\mathrm{Xe}$, which is in contradiction with the Ore model [20] that works for lighter noble gases where spin-orbit effects are small.

Allen Mills (University of California, Riverside, USA) described his experiment with David Cassidy to study manypositron effects at or near the surface of suitably chosen solids [31]. They plan to create $\mathrm{Ps}_{2}$ molecules from Ps atoms trapped in surface states on a metal surface. Since $\mathrm{Ps}_{2}$ decays predominantly by $2 \gamma$ emission, it is expected that the effect can be readily verified by measurement of the ratio of the $3 \gamma$ to $2 \gamma$ rates. A major goal of their work is to create Bosecondensed positronium, a unique, weakly interacting BEC system. As shown in Fig. 6, the signature of a BEC would be a narrow peak in the $2-\gamma$ annihilation spectrum arising from the zero-momentum condensate.

Due to the light mass of Ps, the BEC transition temperature is high, namely $T_{\mathrm{c}} \sim 1500 \mathrm{~K}$ for $n \sim$ $10^{21} \mathrm{~cm}^{-3}$. To create the BEC, they plan to use the scheme proposed earlier by Mills and Platzman [31,32]. This scheme involves focusing a pulsed beam $(\Delta t \leq 100 \mathrm{~ns})$ of $\sim 10^{9} \mathrm{spin}-$ polarized positrons, with energies of a few $\mathrm{keV}$, on the surface of a solid (e.g., silicon) that has a cavity just beneath the surface. The positrons are expected to pick up electrons as they enter the cavity, thus creating the dense Ps gas that is required for a BEC. Challenges include focusing to a small spot on the surface, in order to fill a disk-shaped cavity of dimensions $1 \mu$ diameter by $0.1 \mu$ thick. Another challenge will be to minimize the heating from the incident positron beam. Focusing the beam will require tricks now being developed to manipulate positron plasmas and beams.

In a related talk, Mills also described his ideas about creating stimulated gamma-ray emission using the Ps BEC in a thin, rod-shaped cavity, $0.1 \mu$ in diameter $\times 100 \mu$ long. In this case, after creating the BEC, one would switch the

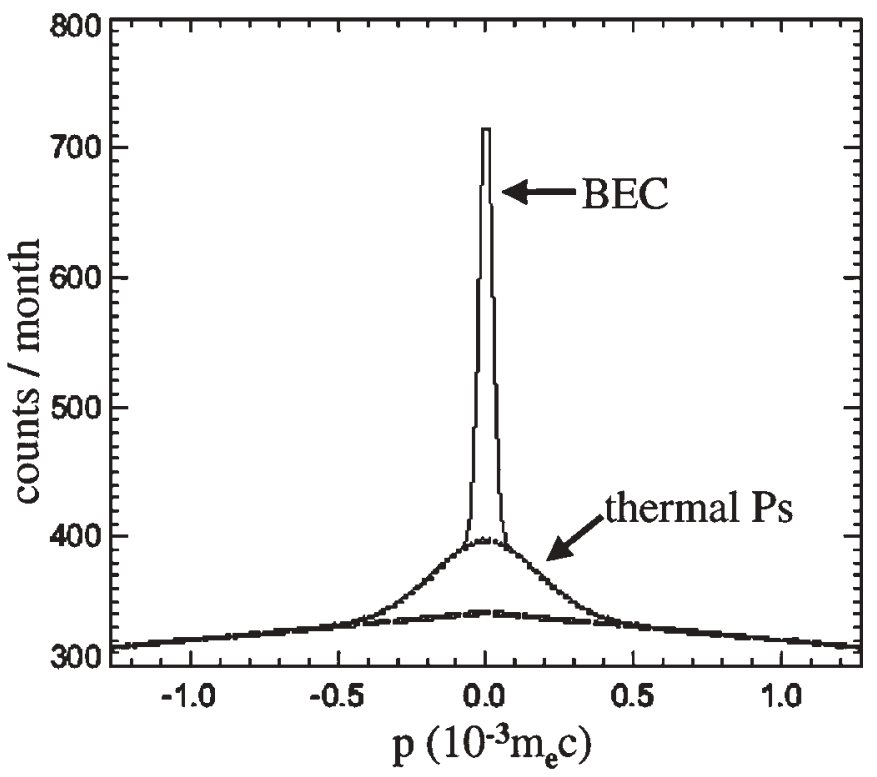

Fig. 6. Simulation of the annihilation spectrum for $10^{8}$ triplet Ps atoms injected in a sub-surface cavity in a solid periodically with a five-minute duty cycle. The calculation is for the spectrum measured using the ACAR technique (angular correlelation of annihilation radiation), with the gamma-ray momenta expressed in terms of the electron rest mass and the speed of light [44]. Shown is the spectrum from $10 \%$ BEC and $90 \%$ thermal Ps (assuming $T=100 \mathrm{~K}$ ), which is superimposed on the background of uncorrelated gamma-ray counts.
Ps atoms from ortho to para to initiate the process of stimulated annihilation [31].

\section{Other topics}

In an after dinner talk, J. Paul (CEA, Saclay, France) presented an overview of positrons in the Universe [1]. There is a strong source of annihilation radiation at our galactic center, localized to approximately the region of the galactic bulge. The source of this emission is believed to be positrons produced by the radioactive decay of nuclei such as ${ }^{56} \mathrm{Co}$. Current measurements are consistent with the majority (i.e., about 93\%) of the annihilation radiation spectrum coming from the decay of Ps. Paul's paper in the conference proceedings presents an overview of this and other positron sources in the Universe.

One of the most important practical applications of positrons is in Positron Emission Tomography (PET), which is used both for diagnostic purposes as well as for research in the function of several organs, especially the brain. A. Gjedde (Aarhus University Hospital, Denmark) discussed the medical applications of PET and prospects for future improvements.

Michael Holzscheiter (Pbar Labs, Newport Beach, USA) described experiments at CERN to evaluate the biological effectiveness of antiproton annihilation for therapy [1]. Of particular interest is the profile of energy deposition of antiprotons in biological samples. Holzscheiter and his collaborators have designed a series of experiments to specifically investigate these effects to determine, for example, whether the energy deposition characteristic of antiprotons is superior to that of protons in therapeutic applications.

N. Oshima (Riken University, Saitama, Japan) described their experiments to accumulate positrons from a ${ }^{22} \mathrm{Na}$ source using Coulomb collisions with an electron plasma as a trapping mechanism [1]. The advantage of this technique is that it is fully UHV compatible. The technique currently is limited to trapping efficiencies $\sim 1 \%$ and accumulation times comparable to the expansion time of the target electron plasma (i.e., $\tau_{\exp } \sim 100 \mathrm{~s}$ ).

\section{Facilities}

One of the main factors limiting present-day positron experiments is the limited fluxes of slow positrons that are currently available. R. Krause-Rehberg (University of Halle, Germany) described plans for the new ELBE positron facility in Rossendorf, Germany. It centers around an electron linac $(40 \mathrm{MeV}$, currents of $1 \mathrm{~mA})$ and has an associated infrared free electron laser (wavelengths $1-200 \mu \mathrm{m})$. The associated positron source will provide a magnetically guided beam with $1 \mathrm{~ns}$ pulses of $\sim 60$ moderated positrons per pulse every $80 \mathrm{~ns}$, corresponding to a slow positron flux approaching $10^{9} \mathrm{~s}^{-1}$. With a magnet behind the sample, the beam-spot size will be $\sim 100 \mu \mathrm{m}$ in diameter. Approximately ten percent of the linac operating time will be for positron experiments.

In a paper in the conference proceedings, R. KrauseRehberg, N. Van der Walt (iThemba LABS, Somerset West, South Africa), and collaborators describe the fabrication of sealed, UHV-compatible ${ }^{22} \mathrm{Na}$ sources for 
low-energy positron experiments [1]. These sealed capsules are now the source of choice in much of the low-energy positron physics community. The design includes a sealed Ti window and is bakeable to $180^{\circ} \mathrm{C}$ and capable of being cooled to cryogenic temperatures (to be compatible with the use of noble-gas moderators).

Christoph Hugenschmidt (Technische Universität München, Garching, Germany) described the design of the new positron source at the Munich fission reactor that is just coming online [1]. The specifications of this exciting new positron user facility include a beam with a flux of $10^{10} \mathrm{~s}^{-1}$ positrons and $2 \mathrm{~cm}$ in diameter. This beam will be tunable from 10 to $3000 \mathrm{eV}$.

I. Meshkov (JINR, Dubna, Russia) described their group's effort to construct a novel and versatile Ps beam source [1]. This device uses a storage ring to collect, cool, and merge positron and electron beams. It is expected to produce a flux of $10^{4}$ ortho-Ps atoms per second at (tunable) energies $\sim 10 \mathrm{keV}$, with a velocity spread $\Delta v / v \sim 10^{-4}$ and an angular divergence $\Delta \theta \sim 1 \mathrm{mrad}$. The corresponding beam decay length is $\sim 8 \mathrm{~m}$. The beam will have $\sim 0.1 \mathrm{eV}$ parallel temperature and $100 \mu \mathrm{V}$ perpendicular temperature. This Ps beam will be important for a range of fundamental physics experiments, including tests of QED.

\section{A look to the future}

Although not addressed directly at the conference, a number of trends in "positron technology" are worthy of note. Trap-based positron beams have recently provided new opportunities for scattering experiments. They now offer improved energy resolution (e.g., $\Delta \varepsilon \sim 20 \mathrm{meV}$ ), and beams with $1 \mathrm{meV}$ energy resolution are under development. Solid noble-gas moderators are now common, offering conversion efficiencies in excess of $1 \%$, which is an improvement of more than an order of magnitude over metallic moderators used previously. While the positron energy spreads are not as small from the noble-gas moderators, they are particularly useful in conjunction with positron traps. This is due to the fact that it is then roughly the temperature of the trapped positrons, and not the moderator, that determines the energy resolution of the resulting positron beam.

Areas that would benefit from further development include more intense positron sources (e.g., the reactor and linac-based beams described above) and positron moderators with even greater efficiency. New methods would be particularly welcome in the development of brighter lowenergy positronium beams for atomic scattering experiments. The conventional technique, namely to create a Ps beam by charge-exchange in a gas cell, has a modest efficiency $\sim 10^{-3}$ and produces a beam with a relatively broad angular distribution. If there is a "magic bullet" for positronium beams, analogous to the development of advanced, trap-based positron beams, this novel idea has yet to surface.

Many interesting collision problems were discussed at the workshop. One example is the positron analog of the $\mathrm{e} \rightarrow 2 \mathrm{e}$ problem (i.e., $A+\mathrm{e}^{+} \rightarrow A^{+}+\mathrm{e}^{+}+\mathrm{e}^{-}$), examining with precision the correlations between the outgoing projectiles. The beginning of work along these lines was reported at the conference by Kover et al. [1]. The challenge for the experimentalists will be to improve the precision and detail of the measurements to be comparable, for example, to what is now done in electron, (e, 2e) experiments.

Another positron problem that is currently of keen interest is the binding of positrons to atoms and molecules. As discussed above, experiments have now established that positrons bind to hydrocarbon molecules with more than $\sim$ two or three carbons. While this phenomenon is understood at some level, a detailed $a b$ initio theoretical treatment is still lacking. On the other hand, it is well established theoretically that positrons bind to a number of atoms, but at present, this lacks experimental test. As a consequence, the topic of positron bound states with atoms and molecules is likely to be one of keen interest in the next few years.

There are a number of potential synergies for the positron community that could be further cultivated. As described above, new facilities are now coming on line that could offer substantial gains in data acquisition rates for scattering experiments. There also continue to be areas of common interest between the collision atomic physics community and areas such as biophysics, surface science, and study of clusters and nanoparticles that would benefit from collaborations between these communities and positron-atomic physicists.

Finally, there is a desire to more fully engage the electron collision community in positron problems. The advent of a new range of precision measurement techniques using positrons and precision comparisons between electron and positron scattering from atomic and molecular targets offer new opportunities for theoretical work. Engaging the larger electron-collision theoretical community would be of enormous help in bringing additional resources and ideas to bear on positron problems. With regard to a closer coupling between the two collision-physics communities, the next positron workshop will be held in conjunction with the next electron-molecule workshop. In particular, the XIIIth International Workshop on Positron and Positronium Physics and the International Symposium on Electron-Molecule Collisions and Swarms will be held in Campos de Jordão, in State of São Paulo, Brazil, July $27-30,2005$. This is an exceedingly welcome development that is likely to foster closer ties between these communities, and it has the potential to spawn a number of new synergetic activities.

\section{Acknowledgements}

We would like to thank the participants of the Workshop for making it an interesting and exciting meeting and for their contributions to the progress of the field. We are especially grateful to Ulrik Uggerhoj for his assistance, and to M. Biasini, G. Laricchia, J. Marler, A. Mills, T. Stein, and D. van der Werf, for providing figures for this paper.

\section{References}

1. Proc. XXIIth International Workshop Positron and Positronium Physics, Sandjberg, Denmark, July 2003; H. Knudsen, T. Ichioka, and U. Uggerhoj, eds., Nucl. Instr. Meth. B, to be published.

2. Amoretti, M. et al., Nature 419, 456 (2002).

3. Gabrielse, G. et al., Phys. Rev. Lett. 89, 233401 (2002). 
4. Gabrielse, G. et al., Phys. Rev. Lett. 89, 213401 (2002).

5. Amoretti, M. et al., private communication (2003).

6. Glinsky, M. E. and O’Neil, T. M., Phys. Fluids B 3, 1279 (1991).

7. Gilson, E. P. and Fajans, J., Phys. Rev. Lett. 90, 015001 (2003).

8. Mohri, A. and Yamazaki, Y., Europhys. Lett. 63, 207 (2003).

9. Hessels, E. A., Homan, D. M. and Cavagnero, M. J., Phys. Rev. A 57, 1668 (1998).

10. Greenberg, O. W., Phys. Rev. Lett. 89, 231602 (2002).

11. Jonsell, S. et al., unpublished.

12. Gilbert, S. J., Kurz, C., Greaves, R. G. and Surko, C. M., Appl. Phys. Lett. 70, 1944 (1997)

13. Gilbert, S. J., Barnes, L. D., Sullivan, J. P. and Surko, C. M., Phys. Rev. Lett. 88, 0443201 (2002)

14. Barnes, L. D., Gilbert, S. J. and Surko, C. M., Phys. Rev. A 67, 032706 (2003)

15. Gribakin, G. F., Phys. Rev. A 61, 22720 (2000).

16. Paul, D. A. L. and Saint-Pierre, L., Phys. Rev. Lett. 11, 493 (1963).

17. Deutsch, M., Phys. Rev. 83, 455 (1951).

18. Sullivan, J. P. et al., Phys. Rev. Lett. 87, 073201 (2001).

19. Goldanskii, V. I. and Sayasov, Y. S., Phys. Lett. 13, 300 (1964).

20. Wright, G. L., Charlton, M., Griffith, T. C. and Heyland, G. R., J. Phys. B 18, 4327 (1985).

21. Murphy, T. J. and Surko, C. M., J. Phys. B 23, L727 (1990).

22. Kernoghan, A. A., McAlinden, M. T. and Walters, H. R. J., J. Phys. B 29, 3971 (1996)

23. Marler, J., unpublished.

24. Laricchia, G., VanReeth, P., Szluinska, M. and Moxom, J., J. Phys. B 35, 2525 (2002).
25. Berakdar, J., Phys. Rev. Lett. 81, 1393 (1998).

26. Garner, A. J., Ozen, A. and Laricchia, G., J. Phys. B 33, 1149 (2000).

27. Blackwood, J. E., McAlinden, M. T. and Walters, H. R. J., J. Phys. B 35, 2661 (2002).

28. Mitroy, J. and Bromley, M. W. J., Phys. Rev. A 67, 034502 (2003).

29. Wheeler, J. A., Ann. New York Acad. Sci. 48, 219 (1946).

30. Mitroy, J. and Novikov, S. A., Phys. Rev. Lett. 90, 183202 (2003).

31. Mills, A. P., Jr. Nucl. Instrum. Meth. B 192, 107 (2002).

32. Platzman, P. M. and Mills, J. P., J. Phys. Rev. B 49, 454 (1994).

33. Barnes, L. D. et al., Physica Scripta in press (2004).

34. Stein, T. S. et al., Hyperfine Interact. 73, 53 (1992).

35. McAlinden, M. T. and Walters, H. R. J., Hyperfine Interact. 73, 65 (1992).

36. Gilmore, S., McAlinden, M. T. and Walters, H. R. J., Unpublished (2003).

37. Garner, A. J., Laricchia, G. and Ozen, A., J. Phys. B 29, 5961 (1996).

38. Zafar, N., Laricchia, G., Charlton, M. and Garner, A. J., Phys. Rev. Lett. 76, 1959 (1996).

39. Skalsey, M. et al., Phys. Rev. A 67, 022504 (2003).

40. Coleman, P. G. et al., J. Phys. B 27, 981 (1994).

41. Blackwood, J. E., McAlinden, M. T. and Walters, H. R. J., J. Phys. B 36, 797 (2003).

42. Biswas, P. K. and Adhikari, S. K., Chem. Phys. Lett. 317, 129 (2000).

43. McAlinden, M. T., MacDonald, F. G. R. S. and Walters, H. R. J., Can. J. Phys. 74, 434 (1996).

44. Basini, M., private communication (2004). 